\title{
The mechanism of partial rupture of a locked megathrust:
}

\section{The role of fault morphology}

\author{
Qiang Qiu ${ }^{1,2}$, Emma M. Hill ${ }^{1,2}$, Sylvain Barbot ${ }^{1,2}$, Judith Hubbard ${ }^{1,2}$, Wanpeng \\ Feng $^{3}$, Eric Lindsey ${ }^{1,4}$, Lujia Feng ${ }^{1}$, Keren Dai ${ }^{5}$, Sergey V. Samsonov ${ }^{6}$, and Paul \\ Tapponnier $^{1,2}$
}

${ }^{1}$ Earth Observatory of Singapore, Nanyang Technological University, Singapore

${ }^{2}$ Asian School of the Environment, Nanyang Technological University, Singapore

${ }^{3}$ Institute of Geophysics, China Earthquake Administration, Beijing, China

${ }^{4}$ Institute for Geophysics and Planetary Physics, University of California, San Diego, USA

${ }^{5}$ Dept. of Remote Sensing and Geospatial Information Engineering, Southwest Jiaotong University, Chengdu, China

${ }^{6}$ Canada Centre for Mapping and Earth Observation, Natural Resources Canada, Canada

\section{Contents of this file}

Text S1, S2

Figures $\mathrm{S} 1$ to $\mathrm{S} 5$

Tables S1

\section{Introduction}

This supplementary information contains details about the dynamic earthquakecycle modeling procedure (Text S1); dynamic model assumptions and limitations (Text S2); the combined multiple tracks of InSAR data used in the study (Table S1); and forward model prediction and residuals between the data and the forward model prediction based on our geodetic inversion modeling (Figure S1 to S4); and the Coulomb stress changes on the megathrust from our preferred slip model (Figure S5).

\section{Text S1.}

We simulate the slip evolution on an isolated segment of the Main Himalayan Thrust (MHT) using a rate-and-state friction framework. We employ the boundary integral method with radiation damping (Rice et al., 2001; Kato, 2003). As with the inversion, we discretize the geometry of the MHT using triangular elements. We obtain the stress kernels using the analytic formulation of Nikkhoo and Walter (2015). We use uniform friction properties with the static friction coefficient $\mu_{0}=0.6$ at steady state with the reference velocity $\mathrm{V}_{0}=10^{-6} \mathrm{~m} / \mathrm{s}, \mathrm{a}=\mathrm{d} \mu / \mathrm{d}(\ln \mathrm{V})=10^{-2}$, the effective confining pressure 
$\sigma=300 \mathrm{MPa}$, and the characteristic weakening distance $\mathrm{L}=7.5 \mathrm{~cm}(\mu$ is the frictional resistance and $\mathrm{V}$ is the slip velocity).

We define the region that could potentially rupture as an area larger than the rupture patch of the $2015 \mathrm{M}_{\mathrm{w}} 7.8$ Gorkha earthquake, extending from the surface to 20 $\mathrm{km}$ depth and about the length of the Kathmandu Klippe (Figure 2B). This region is modeled with velocity-weakening properties, with $(a-b)=d \mu_{s s} / d(\ln V)=-4 \times 10^{-3}$, where $\mu_{\mathrm{ss}}$ is the frictional resistance at steady state. Outside this region, the fault is modeled with the velocity-strengthening properties of $(a-b)=4 \times 10^{-3}$. We load the fault at a uniform and constant rate of $\mathrm{V}_{\mathrm{pl}}=0.02 \mathrm{~m} / \mathrm{yr}$.

In the simulation, we obtain a series of earthquakes of varying magnitudes and with ruptures covering sometimes a fraction of the velocity-weakening patch and some other times the entirety of the patch. The complexity of the slip history emerges spontaneously from the dynamics of the system. In the context of our simulation, the complexity of the slip history comes from the morphology of the megathrust, not from heterogeneous initial conditions or spatial distribution of friction properties. (If the fault were perfectly planar, such a distribution of frictional properties would lead to a single type of earthquakes with a characteristic size and a single recurrence time and both recurrence times and rupture slip would be predictable.) The simulated fault history contains events with characteristics that resemble those of the $2015 \mathrm{M}_{\mathrm{w}} 7.8$ Gorkha earthquake. The Gorkha-like ruptures extend through the deep décollement but are confined by the two ramps. Other single-segment ruptures occupy the upper décollement or the topmost ramp, resulting in earthquakes of $\mathrm{M}_{\mathrm{w}}<8$. Occasionally, ruptures spread from the surface to the bottom of the seismogenic zone in a through-going, multi-segment event, resulting in a great earthquake with $\mathrm{M}_{\mathrm{w}}>=8$. The simulation is only intended to explain the role of geometry on the complexity of earthquake dynamics in the context of a long sequence of earthquakes. In reality, strong heterogeneities in composition, rheology and other physical properties make the earthquake history even more complex. As we present a simulation with a simple distribution of dynamic frictional properties, some important differences with the Gorkha setting arise. For example, the simulated afterslip that follows the $\mathrm{M}_{\mathrm{w}} 7.7$ event, which is similar to the Gorkha earthquake, has hundreds $\mathrm{cm}$ of afterslip downdip of the coseismic rupture, presumably more than expected for this event.

In this study, we highlight one particular combination of effective normal stress and dynamic friction properties to show that fault morphological barriers can control the extent of earthquake ruptures and introduce complexity in earthquake cycles. Admittedly, not all distributions of dynamic friction properties produce similar results. Our study merely provides a proof of existence of a combination of frictional parameters and geometrical properties spontaneously giving rise to segmentation. We do not aim to reproduce every aspect of the 2015 Gorkha event. Theoretical insight about the propagation of elasto-dynamic rupture across fault bends can be found in the work of Poliakov et al. (2002) and Rice et al. (2005).

\section{Text S2.}


We model earthquake cycles using the rate-and-state framework and the boundary integral method under the radiation-damping approximation. The method allows us to evaluate earthquake cycles on geometrically complex, non-planar, faults. This is accomplished by damping of the seismic waves, ignoring the stress concentration at the rupture tip induced by the wave-mediated stress transfer. The numerical simulation might produce biased estimates of rupture size, recurrence times, and other rupture characteristics (Thomas et al., 2014). 


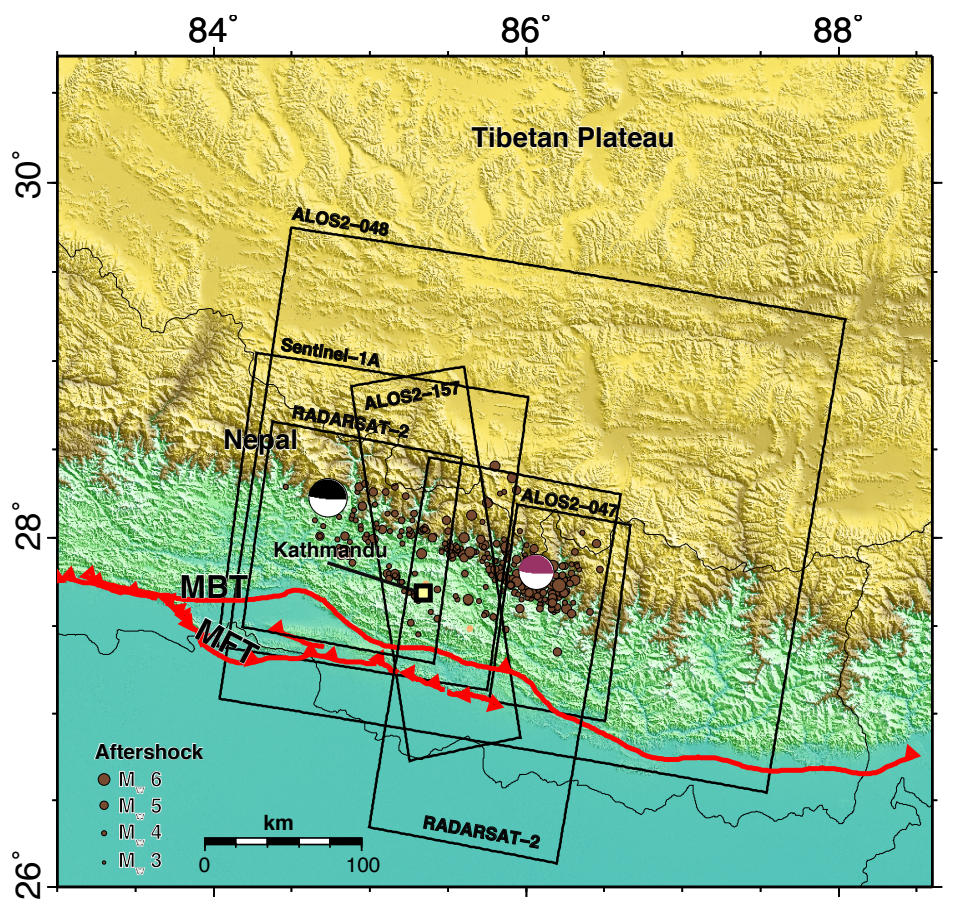

Figure S1. Footprint of InSAR data considered in this study, including from the Sentinel1A, RADARSAT-2, and ALOS-2 satellites. The beach balls represent the moment tensors of the mainshock and largest aftershock. The coloured circles correspond to aftershocks (same as in Figure 1). 

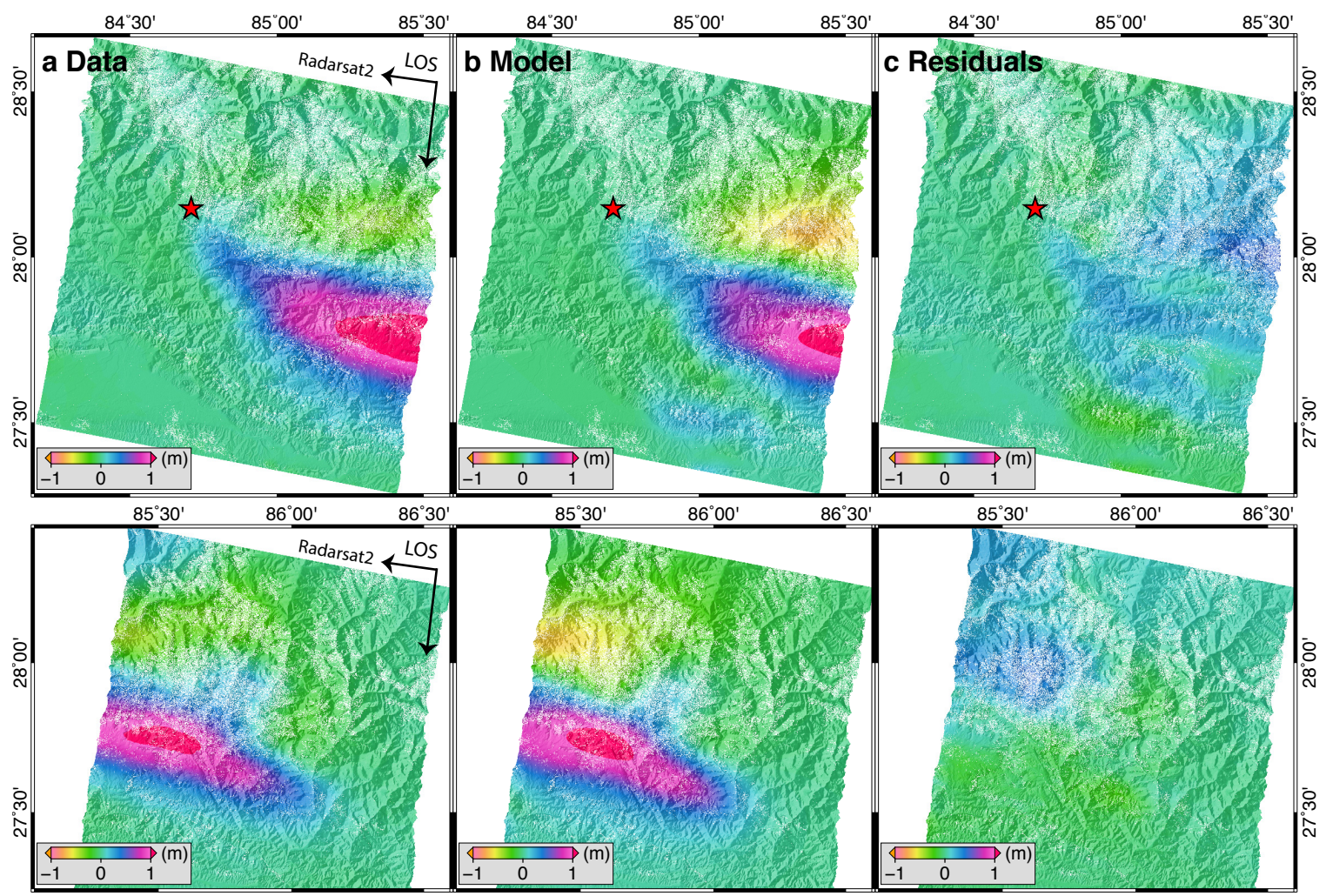

Top panel 

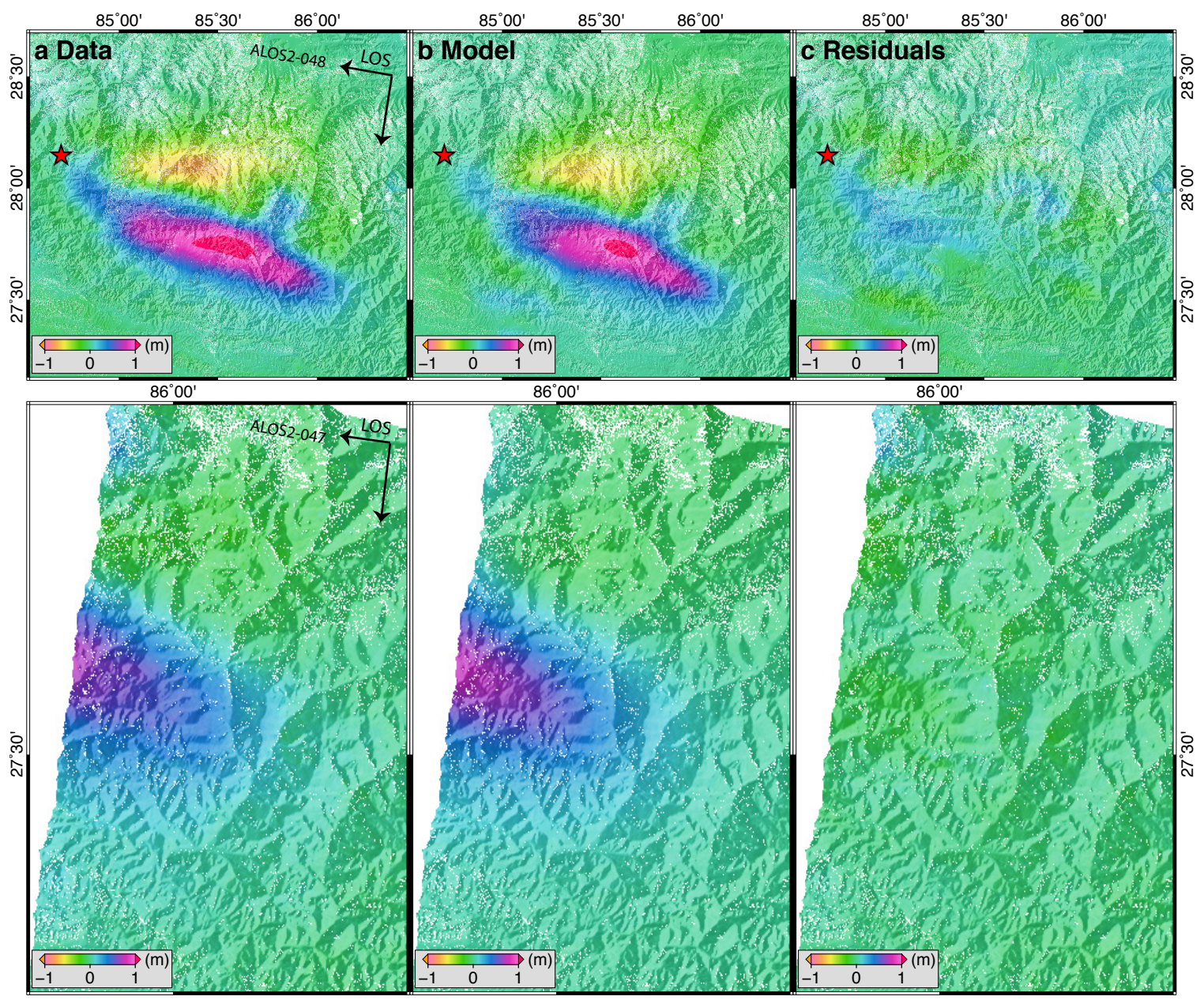

Second panel 


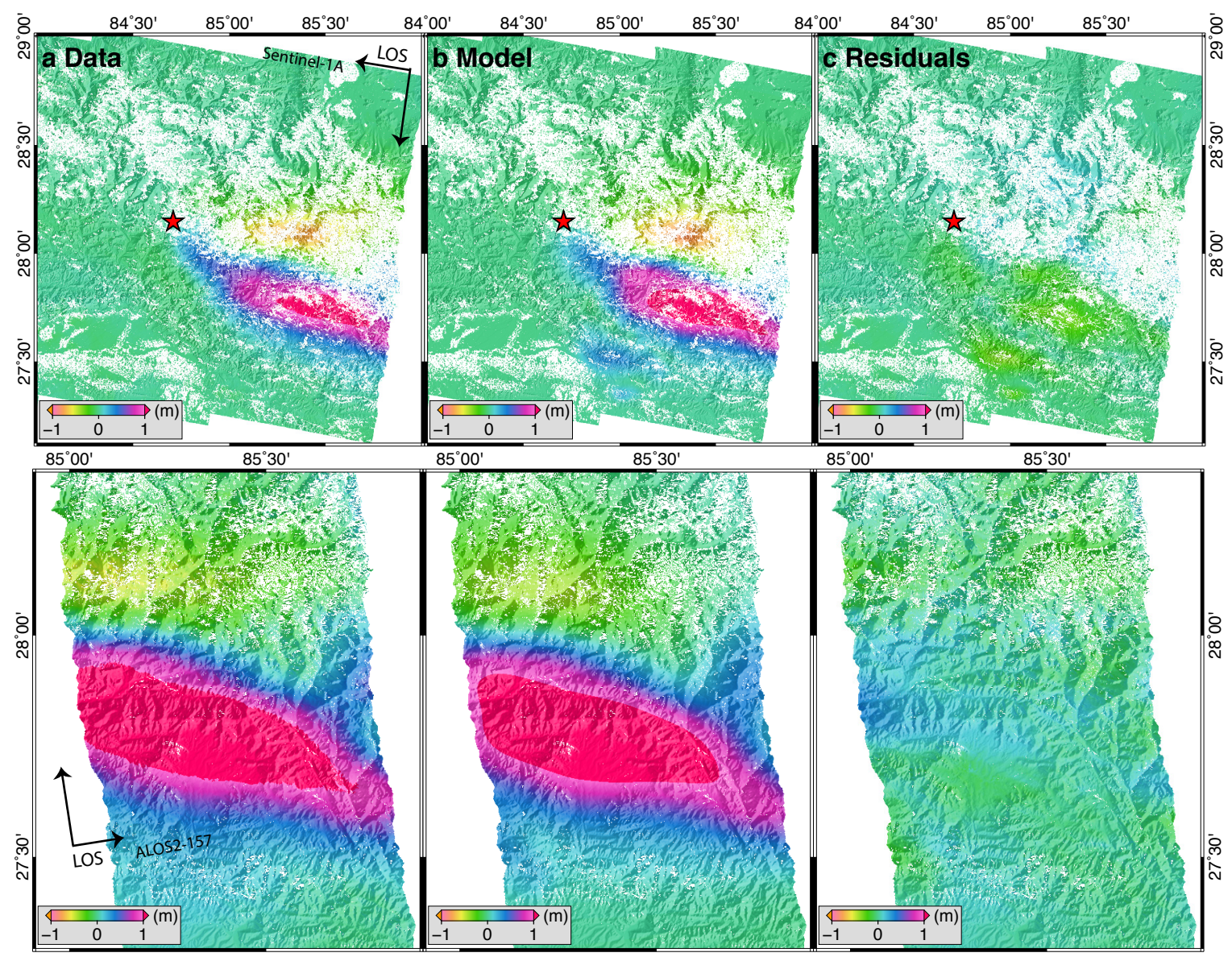

Bottom panel

Figure S2. InSAR data (left), model (middle) and residuals (right). Top panel is for RADARSAR-2 data, second panel is for ALOS-2 tracks 048 and 047. Bottom panel is for Sentinel-1A, and ALOS2-track 157. 


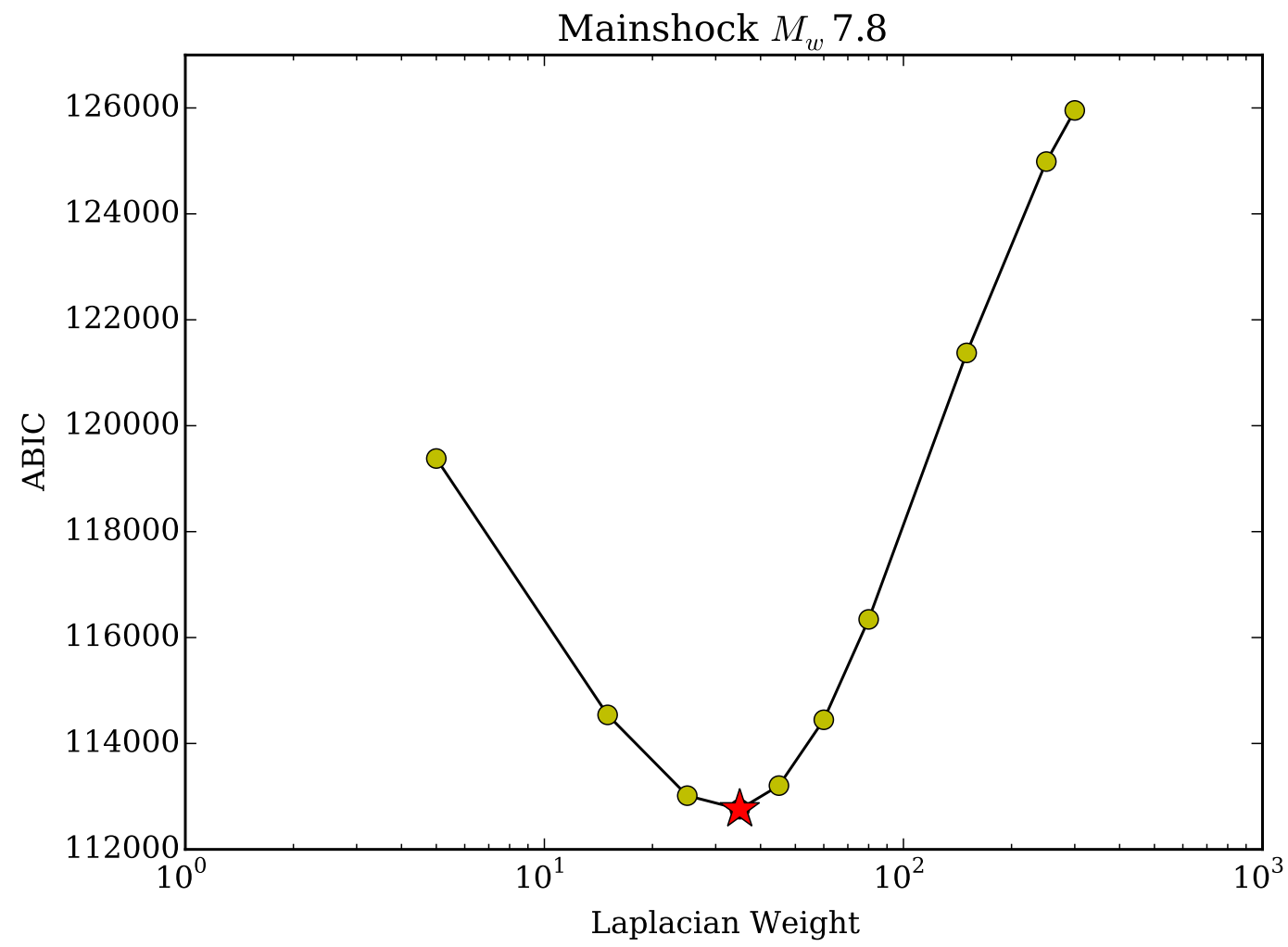

Figure S3. The Lapacian weight for spatial smoothing in the inversion for slip was selected using Akaike's Bayesian Information Criterion (Yabuki and Matsuura, 1992). The red star indicates the Laplacian weight used for our preferred model. 

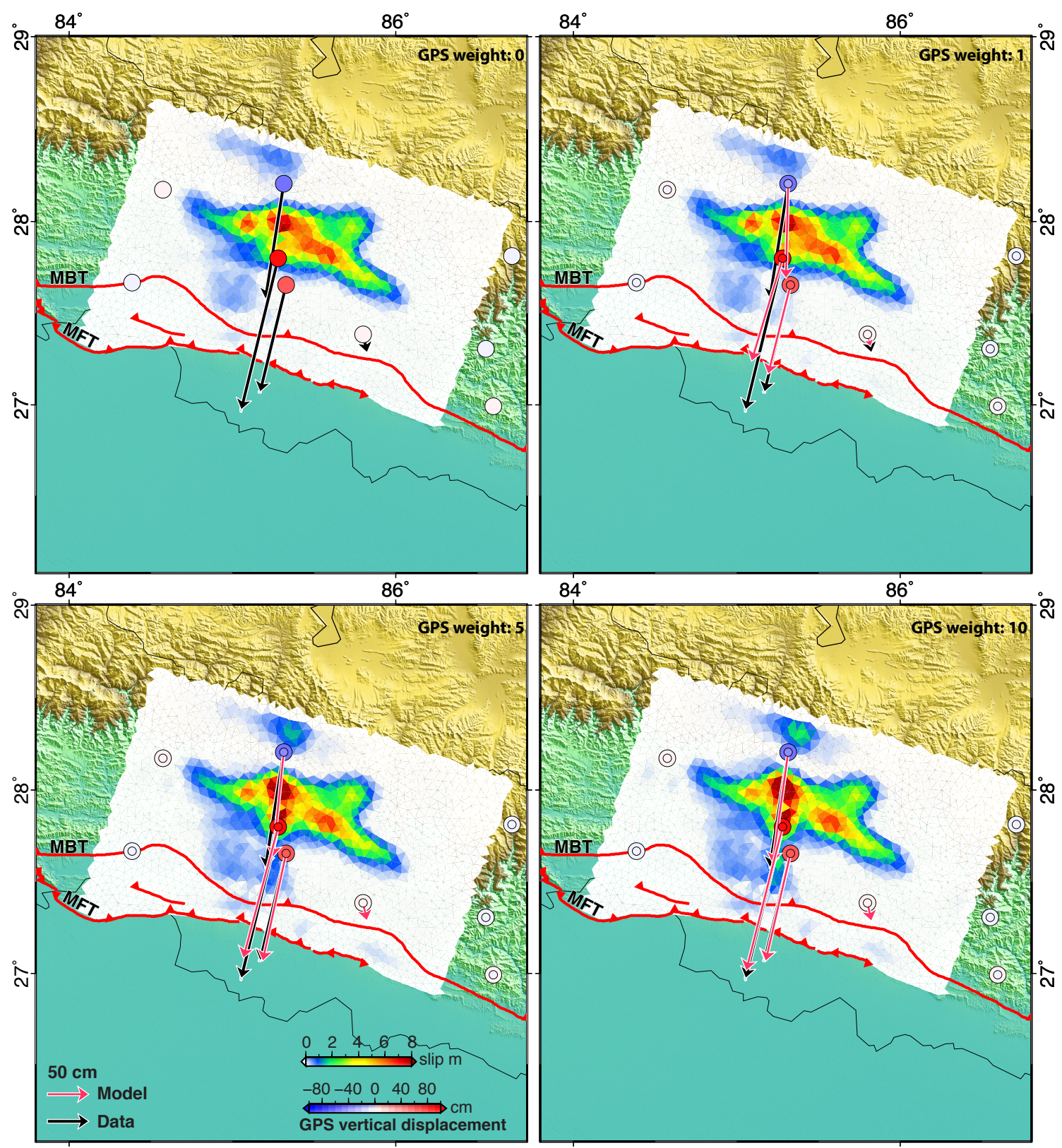

Figure S4. Examples of estimated slip distributions obtained using different relative weights for data input to the slip inversion. The GPS data prefer a little more shallow slip, but in general the distributions remain similar regardless of data weight. 


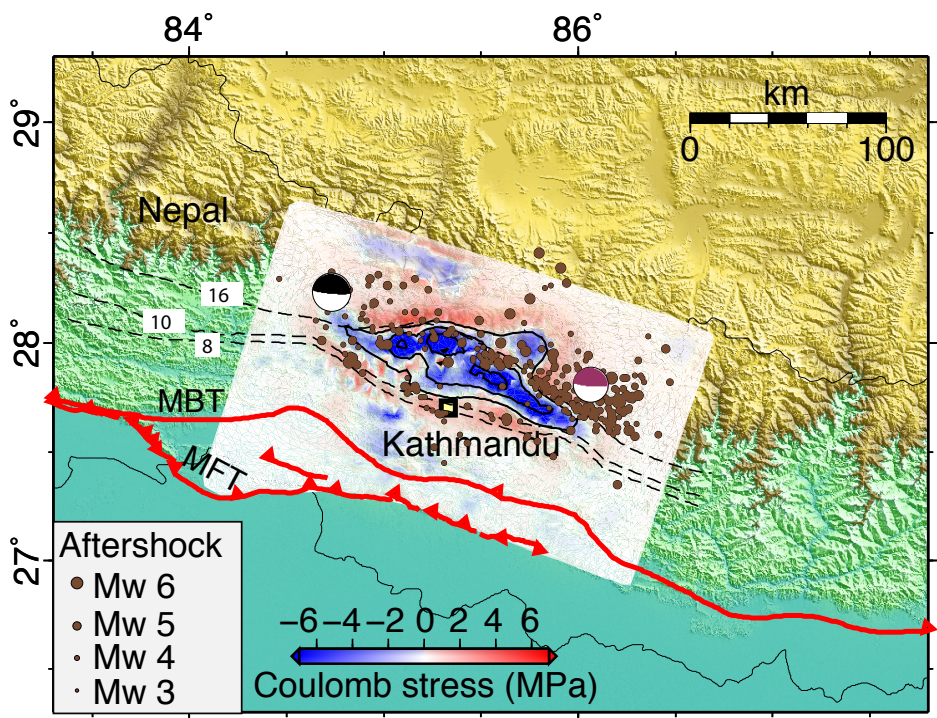

Figure S5: Coulomb stress changes on the megathrust from our preferred slip model, assuming a coefficient of friction of $\mu_{0}=0.6$. The dash lines show the location of the shallow $(8-10 \mathrm{~km})$ and deep ramp $(16 \mathrm{~km})$, respectively. The coloured dots correspond to aftershocks (same as Figure 1). The beach balls represent the moment tensor of the mainshock and largest aftershock.

Table S1. Details of the InSAR data used in this study.

\begin{tabular}{|c|c|c|c|c|c|c|}
\hline $\operatorname{Inf}$ & Mission $^{\mathrm{a}}$ & Mode & $\begin{array}{c}\text { Flight } \\
\text { Direction }\end{array}$ & Track & Master & Slave \\
\hline 1 & ALOS-2 & ScanSAR & DSC & T047 & 20150331 & 20150428 \\
\hline 2 & ALOS-2 & ScanSAR & DSC & T048 & 20150222 & 20150503 \\
\hline 3 & ALOS-2 & StripMap & ASC & $\mathrm{T} 157$ & 20150221 & 20150502 \\
\hline 4 & S1A & TOPS & DSC & - & 20150417 & 20150429 \\
\hline 5 & $\mathrm{RS} 2$ & StripMap & DSC & - & 20150405 & 20150429 \\
\hline 6 & RS2 & StripMap & DSC & - & 20150412 & 20150506 \\
\hline
\end{tabular}

\section{Reference:}

Kato, N., 2003, Repeating slip events at a circular asperity: Numerical simulation with a rate- and state- dependent friction law: Bull. Earthq. Res. Inst., v. 78, p. 151-166.

Nikkhoo, M., and Walter, T.R., 2015, Triangular dislocation: an analytical, artefact-free solution: Geophys. J. Int., v. 201, no. 2, p. 1119-1141, doi: 10.1093/gji/ggv035. 
Poliakov, A.N.B., Dmowska, R., and Rice, J.R., 2002, Dynamic shear rupture interactions with fault bends and off-axis secondary faulting: Journal of Geophysical Research: Solid Earth, v. 107, no. B11, p. ESE 6-1-ESE 6-18, doi: 10.1029/2001JB000572.

Rice, J.R., Lapusta, N., and Ranjith, K., 2001, Rate and state dependent friction and the stability of sliding between elastically deformable solids: J. Mechan and Physics of Solids, v. 49, no. 9, p. 1865-1898, doi: 10.1016/S0022-5096(01)00042-4.

Rice, J.R., Sammis, C.G., and Parsons, R., 2005, Off-Fault Secondary Failure Induced by a Dynamic Slip Pulse: Bulletin of the Seismological Society of America, v. 95, no. 1, p. 109-134, doi: 10.1785/0120030166.

Thomas, M.Y., Lapusta, N., Noda, H., and Avouac, J.-P., 2014, Quasi-dynamic versus fully dynamic simulations of earthquakes and aseismic slip with and without enhanced coseismic weakening: Journal of Geophysical Research: Solid Earth, v. 119, no. 3, p. 1986-2004, doi: 10.1002/2013JB010615. 